\title{
Erratum to: A Bernstein Property of a Class of Fourth Order Complex Partial Differential Equations
}

\author{
Gang Chen and Li Sheng
}

Erratum to: Results. Math. DOI 10.1007/s00025-010-0030-8

There is a misprint in our paper.

In Sect. 5 in the sentence "Without loss of generality, we can assume $\Phi^{c}\left(p^{*}\right) \geq 1$. In fact if $\Phi^{c}\left(p^{*}\right) \leq 1 \ldots$. should be "Without loss of generality, we can assume $\Phi^{c}\left(p^{*}\right)>0$. In fact if $\Phi^{c}\left(p^{*}\right)=0 \ldots$.

Gang Chen and Li Sheng

Department of Mathematics

Sichuan University

Chengdu 610064

People's Republic of China

e-mail: bzongbzongbzong@163.com;

1_sheng@yahoo.cn

The online version of the original article can be found under doi:10.1007/s00025-010-0030-8. 